\title{
Geometric Methods in the Quantum Many-Body Problem. Nonexistence of Very Negative Ions ${ }^{\star}$
}

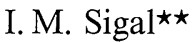 \\ The Weizmann Institute of Science, \\ Department of Theoretical Mathematics, Rehovot 76100, Israel
}

\begin{abstract}
In this paper we develop the geometric methods in the spectral theory of many-body Schrödinger operators. We give different simplified proofs of many of the basic results of the theory. We prove that there are no very negative ions in Quantum Mechanics.
\end{abstract}

\section{Introduction}

In this paper we develop geometric methods for studying the spectral properties of the many-body Schrödinger operators. The adjective "geometric" refers to the basic role played by the analysis of the space configurations of a many-body system in question. To translate this geometry into the quantum-mechanical language, one uses partitions of unity on the state space, $L^{2}$ (configuration space). This approach commands a remarkable flexibility. As with trial functions in the variational principle, one can vary and optimize the partitions of unity depending on a problem at hand. This will be demonstrated in the present paper. Moreover, the method is naturally generalizable to operators on manifolds.

The basic property of the Schrödinger operators which permits such an analysis is their locality. Nevertheless, it is remarkable that basically local methods give detailed information about the spectra which are the global characteristics of operators.

The geometric methods in the many-body QM problem first appeared in the pioneering work of Zhislin [Z1]. In the West the geometric ideas come from the works of R. Haag and D. Ruelle on the scattering in the field theory and of LaxPhillips on the acoustic scattering. The term "geometric methods" was dubbed by B. Simon [Sim1]. The importance of partitions of unity in the QM many-body problem was realized in the classical works of Enss [E] and Simon [Sim1] (see also Deift and Simon [DS]). (For more complete references and detailed comments see $[\mathrm{RS} 3]$.)

* Partially supported by USNSF Grant MCS-78-01885

$\star \star$ Charles H. Revson Senior Scientist 
In this paper we discuss the following topics:

Section 2: Hunziker-van Winter-Zhislin (HVZ) theorem (warm up).

Section 3: Exponential fall-off of eigenfunctions.

Section 4: Number of bound states. Short-range systems and negative ions.

Section 5: Nonexistence of very negative ions.

In Sects. 2-4 we present simplified proofs of known results, while the result of Sect. 5 is new. Note that Ruskai [R] has earlier, and independently, obtained a similar but slightly weaker result (namely that the ground state energy of a negative ion is bounded from below by a constant ${ }^{1}$ ). All the neccessary definitions are given in Sect. 1 and some of the technical results are carried out into the appendices. Discussions of related results and extensions are presented under the title "Remarks" at the end of the paper. Finally we admit that in estimation we always favored short cuts whatever the price. Probably many of the estimates we use can be improved to give physically interesting results. An announcement of this paper appears in [S3].

\section{Hamiltonians}

The configuration space of an $N$-body system in $\mathbb{R}^{v}$ with masses $m_{1}, \ldots, m_{N}$ in the center-of-mass frame is the hyperplane $X=\left\{x \in \mathbb{R}^{v N} \mid \Sigma m_{i} x_{i}=0\right\}$. We equip $X$ with the inner product [SS] $\langle x, y\rangle=2 \Sigma m_{i} x_{i} y_{i}$. We assume $v \geqq 2$.

The potentials $V_{l}: \mathbb{R}^{v} \rightarrow \mathbb{R}$ are supposed to be Laplacian-compact, i.e. compact as multiplication operators from the Sobolev space $H_{2}\left(\mathbb{R}^{v}\right)$ to $L^{2}\left(\mathbb{R}^{v}\right)$. By the same letter $V_{l}$ we denote also the multiplication operator by $V_{l}\left(x^{l}\right)$ on $L^{2}(X)$. Here $x^{l}=x_{i}-x_{j}$ for $l=(i j)$.

The Schrödinger operator for an $N$-body system in question in the center-ofmass frame is

$$
H=-\Delta+\Sigma V_{l} \text { on } L^{2}(X),
$$

where $\Delta$ is the Laplacian on $X$.

The partitions $a=\left\{C_{i}\right\}$ of the set $\{1, \ldots, N\}$ are collections of nonintersecting subsets $C_{i}$, called below the clusters, of which the union is entire $\{1, \ldots, N\}$.

With each $a$ we associate the intercluster interaction $I_{a}=\sum_{l \neq a} V_{l}$, truncated Hamiltonian $H_{a}=H-I_{a}$ and intercluster distance $|x|_{a}=\min _{l \neq a}\left|x^{l}\right|$. Here $l \$ a$ signifies that the indices of $l$ belong to different clusters of $a$.

A partition of unity on $L^{2}(X)$ is a collection $\left\{X_{i}\right\}$ of (positive) $C^{2}$-functions such that $\Sigma X_{i}^{2}=1$. (Note the difference with the standard definition.)

A localization formula (implicity [C, I, K, M, MS]). Let $\left\{X_{i}\right\}$ be a partition of unity on $L^{2}(X)$. Then

$$
H=\Sigma X_{i} H X_{i}-\Sigma\left|\nabla X_{i}\right|^{2} \text {. }
$$

Proof. In the identity $H=\Sigma X_{i}^{2} H$ commute $X_{i}$ one step to the right and use $\Sigma X_{i} \nabla X_{i}=0$ and $\Sigma\left|\nabla X_{i}\right|^{2}+\Sigma X_{i} \Delta X_{i}=0$ to transform $\Sigma X_{i}\left[X_{i}, H\right]$.

The local partition $a(x)$ is defined by putting $i$ and $j$ into the same cluster of $a(x)$ iff $x_{i}=x_{j}$.

1 Ruskai proved also nonexistence of very negative ions with the electrons replaced by bosons 


\section{HVZ Theorem, Difficult Direction}

The HVZ theorem states $([\mathrm{H}, \mathrm{VW}, \mathrm{Z} 1])$ that $\sigma_{\text {ess }}(H)=\bigcup \sigma\left(H_{a}\right)$. The inclusion $\bigcup \sigma\left(H_{a}\right) \subset \sigma_{\text {ess }}(H)$, called normally the easy direction, is proven by an explicit construction of an approximate eigenfunction for each $\lambda \in \bigcup \sigma\left(H_{a}\right)$ and applying Weyl's criterion [RSI]. We prove here the inclusion

called the difficult direction.

$$
\sigma_{\text {ess }}(H) \subset \bigcup \sigma\left(H_{a}\right)
$$

First, we introduce.

A Ruelle-Simon partition of unity $\left\{J_{a}\right\}$, a runs through all and only two-cluster partitions, is defined by $\operatorname{supp} J_{a}=\left\{\left.x \in X|| x\right|_{a}>d|x|\right\}$ for some number $d$ depending only on $N$ and such that the regions on the right hand side cover $X$. Such $d$ exist by the Ruelle-Simon lemma ([Sim1]). This lemma and a standard construction (e.g. like the one used in the appendix) imply the existence of $\left\{J_{a}\right\}$.

In this paper we do not use directly the definition of $\left\{J_{a}\right\}$. Instead we use

The main property of $\left\{J_{a}\right\}([\operatorname{Sim} 1, \mathrm{RS} 3])$ :

$\left|\nabla J_{a}\right|^{n}$ and $I_{a} J_{a}^{n}, n>0$, are $\Delta$-compact (in fact, they decay in $X$ as $|x|^{-n}$ and the worst potential in $I_{a}$, respectively).

The localization formula with $\left\{J_{a}\right\}$ reads

$$
H=\Sigma\left(J_{a} H J_{a}-\left|\nabla J_{a}\right|^{2}\right) .
$$

Proof. Since $\Sigma\left(I_{a} J_{a}^{2}-\left|\nabla J_{a}\right|^{2}\right)$ is $\Delta$-compact, Weyl's theorem [RS3] yields $\sigma_{\text {ess }}(H)=\sigma_{\text {ess }}\left(\sum J_{a} H_{a} J_{a}\right)$. Furthermore, $\sum J_{a} H_{a} J_{a} \geqq \min \left(\inf H_{a}\right)$. Hence $\sigma\left(\sum J_{a} H_{a} J_{a}\right) \subset \bigcup \sigma\left(H_{a}\right)$ (remember that the spectra of the $H_{a}$ fill entire semiaxes since $H_{a}$ are translationary invariant). Both relations give (2.1).

\section{Exponential Fall-Off of Eigenfunctions}

Theorem [DHSV]. Let $\psi$ be an eigenfunction of $H$ corresponding to an isolated eigenvalue E. Then

$e^{f} \psi \in L^{2}(X) \quad$ as long as $\forall a,|\nabla f|^{2}<\inf H_{a}-E \quad$ in a vicinity of $\{x \in X \mid a(x)=a\}$.

Here we need another partition of unity.

A partition of unity $\left\{X_{a}\right\}$, a runs through all partitions, is defined with respect to the subspaces ([SS, DHSV, A]), $\{x \in X \mid a(x)=a\} \forall a$. $X_{a}$ with two-cluster $a$ live in a neighborhood of $\{x \in X \mid a(x)=a\}$ and so on.

Proof. By virtue of the Combes-Thomas argument (in the DHSV-form) (see [DHSV, CT, RS3] and Appendix 1) it suffices to show that

$$
\operatorname{Inf} \operatorname{Re} \sigma_{\text {ess }}(H(i \lambda f))>E \text { for all } 0 \leqq \lambda \leqq 1
$$

as long as $f$ is restricted as in the theorem. Here $H(f)=(-i \nabla-\nabla f)^{2}+\Sigma V_{l}$. By the abstract inequality (see Appendix 2, cf. [A]),

$$
\inf \operatorname{Re} \sigma_{\text {ess }}(A) \geqq \inf \sigma_{\text {ess }}(\operatorname{Re} A), \quad \text { where } \quad \operatorname{Re} A=\frac{1}{2}\left(A+A^{*}\right),
$$


we find that $\inf \operatorname{Re} \sigma_{\text {ess }}(H(i f)) \geqq \inf \sigma_{\text {ess }}\left(H-|\nabla f|^{2}\right)$. Applying the proof of Sect. 2 with $\left\{J_{a}\right\}$ replaced by $\left\{X_{a}\right\}$ to $H-|\nabla f|^{2}$ we arrive at $\sigma_{\text {ess }}\left(H-|\nabla f|^{2}\right)$ $C \bigcup \sigma\left(H_{a}-|\nabla f|^{2} X_{a}^{2}\right)$. Together with the previous inequality this gives inf $\operatorname{Re} \sigma_{\text {ess }}(H(i f)) \geqq \min _{a} \inf \left(H_{a}-|\nabla f|^{2} X_{a}^{2}\right)$ which implies the desired inequality.

\section{Finiteness of the Number of Bound States}

Theorem 4.1. (Zhislin et al.) The number of bound states of $N$-body short-range (more exactly $V^{l} \in L^{p}\left(\mathbb{R}^{v}\right), p=v / 2$ ) systems whose bottoms of continuous spectra are defined only by two-cluster breakups (i.e. $\inf H_{a}>\inf \sigma_{\text {ess }}(H)$ if a has more than two clusters) is finite.

Theorem 4.2. [Yafaev (3 particles), Zhislin, Zhislin-Vugalter]. Negative ions can have only a finite number of bound states.

What makes the last theorem true is the QM Newton screening (by $N-1$ electrons, in the ground state, of the nucleus of charge $Z \leqq N-1$ from the other electron). If the potentials are changed in such a way that the ground states of the $(N-1)$-electron system have different parities or do not have definite parities at all [which might happen if there is an additional ("accidental") degeneracy], then the effective potential between this system and the other electron has a dipole leading term $\left(|x|^{-2}\right.$ at infinity). This term when sufficiently large might lead to the infinite number of bound states for $H$.

Now we explain the main ideas of the proof of the theorems. First we transform $H$ using the localization formula with the partition of unity $\left\{X_{a}\right\}$ defined in Sect. 3:

$$
\begin{aligned}
H & =\Sigma\left(X_{a} H X_{a}-\left|\nabla X_{a}\right|^{2}\right) \\
& =\Sigma\left(X_{a} K_{a} X_{a}-\left|\nabla X_{a}\right|^{2}\right),
\end{aligned}
$$

where $K_{a}=H_{a}+I_{a} \tilde{X}_{a}$ and $\tilde{X}_{a}=1$ on $\operatorname{supp} X_{a}$. Here we have used that $\tilde{X}_{a} X_{a}=X_{a}$. This step is where the geometric methods enter changing radically the problem: $I_{a} \tilde{X}_{a}$ behaves as a multiparticle potential, namely, it vanishes in all directions, provided we choose $\operatorname{supp} \tilde{X}_{a} \subset\left\{\left.x \in X|| x\right|_{a}>\tilde{d}|x|\right\}$.

Next we note that the operators $K_{a}$ with $a$ such that $\inf H_{a}>\Sigma$, where $\Sigma=\inf \sigma_{\text {ess }}(H)$, contribute only a finite number of eigenvalues $<\Sigma$. For the operators $K_{a}$ with $a$ such that $\inf H_{a}=\Sigma$ we use the Combes-Simon inequality $A \geqq P A P-\delta^{-1} P A Q A P+Q(A-\delta) Q, \delta>0$, to decouple the part along $P_{a} L^{2}(X)$ from the part along $Q_{a} L^{2}(X)$. Here $P_{a}=$ [projection on the eigenspace of $H^{a}$ corresponding to $\left.\inf H^{a}=\inf \left(H_{a}\right)\right] \otimes \mathbb{1}$ and $Q_{a}=\mathbb{1}-P_{a}$ with $H^{a}$ the operator obtained from $H_{a}$ after removal of the center-of-mass motion of the clusters in $a$. The resulting operators on $Q_{a} L^{2}(X)$ have inf (essential spectrum) $>\Sigma$, so they contribute again only finite numbers of eigenvalues $<\Sigma$. The operators on $P_{a} L^{2}(X)$ are one-particle Schrödinger operators with the two-cluster effective potentials. Finally one takes care of the error term $\Sigma\left|\nabla X_{a}\right|^{2}$ using the fact that it lives in the region of $X$ where the system in question splits into three or more clusters. Collecting all the estimates above we observe that $H \geqq \Sigma X_{a} F_{a} X_{a}$, where each $F_{a}$ has only a finite number of eigenvalues $<\Sigma$. Hence so have $\Sigma X_{a} F_{a} X_{a}$ and, by the comparison theorem, $H$. 
We deduce Theorems 4.1 and 4.2 from a general result given below. It is a slight generalization of the results of Zhislin and his collaborators [Z2, Z3, AZS, ZV].

Recall that $H^{a}$ is the operator obtained from $H_{a}$ after the removal of the centerof-mass motion of the clusters in $a$. The ground states, $\psi^{a}$, of the $H^{a}$ are nondegenerate ([Zh4, RS3]). This is not, in general, true if one restricts $H^{a}$ to functions of a certain symmetry type. However, we assume for the sake of notations that these ground states are nondegenerate too.

Theorem 4.3. (Essentially Zhislin et al.) Assume that inf $\sigma_{\text {ess }}(H)$ is defined only by two-cluster breakups (i.e. $\inf H_{a}>\inf \sigma_{\text {ess }}(H)$ for all three-cluster decompositions a) and assume that for any two-cluster partition $a$ with $\inf H^{a}=\inf \sigma_{\text {ess }}(H)$ the one-body hamiltonian $-\Delta+W_{a}\left(\right.$ on $L^{2}\left(\mathbb{R}^{v}\right)$ ), where

$$
W_{a} \equiv\left\langle\psi^{a}, I_{a} \psi^{a}\right\rangle-\delta^{-1}\left(\left\langle\psi^{a}, I_{a}^{2} \psi^{a}\right\rangle-\left\langle\psi^{a}, I_{a} \psi^{a}\right\rangle^{2}\right), \quad \delta>0,
$$

has only a finite number of bound states. Then the discrete spectrum of $H$ is finite. The analogous result holds also on the subspaces of functions of definite symmetry types.

Proof. Denote by \# $(a)$ the number of clusters in a partition $a$. Set, as usual, $\langle x\rangle=\left(1+|x|^{2}\right)^{+1 / 2}$. Let $\tilde{W}_{a}$ is obtained from $W_{a}$ by the substitution $I_{a} \rightarrow I_{a} \tilde{X}_{a}$.

Lemma 4.4. For any $\delta>0$ and $\varepsilon>0$ the following inequality holds

$$
H \geqq \sum_{\neq(a)=2} X_{a} B_{a} X_{a}+\sum_{\neq(a) \geqq 3} X_{a}\left(H_{a}+I_{a} \tilde{X}_{a}+C_{\varepsilon}\langle x\rangle^{-2}\right) X_{a},
$$

where $B_{a}=C_{a} \oplus D_{a}$ on $P_{a} L^{2}(X) \oplus Q_{a} L^{2}(x)$ with

and

$$
C_{a}=-\Delta+\tilde{W}_{a}-\varepsilon\langle x\rangle^{-2}-\Sigma \text { on } L^{2}\left(\mathbb{R}^{v}\right)
$$

$$
D_{a}=Q_{a}\left(H_{a}-\delta+I_{a} \tilde{X}_{a}-\varepsilon\langle x\rangle^{-2}\right) Q_{a} .
$$

Proof. We apply the Combes-Simon decoupling inequality [Sim1]

$$
K_{a} \geqq P_{a} K_{a} P_{a}-\delta^{-1} P_{a} K_{a} Q_{a} K_{a} P_{a}+Q_{a}\left(K_{a}-\delta\right) Q_{a},
$$

obtained by applying the Schwartz inequality to $P_{a} K_{a} Q_{a}+Q_{a} K_{a} P_{a}$ [see Remark (a) to this section], to each $K_{a}$ with $\#(a)=2$ on the right hand side of (4.1).

Next, we estimate the localization error as

$$
\Sigma\left|\nabla X_{a}\right|^{2} \leqq\left(\varepsilon \sum_{\#(a)=2} X_{a}^{2}+C_{\varepsilon} \sum_{\#(a) \geqq 3} X_{a}^{2}\right)\langle x\rangle^{-2},
$$

for any $\varepsilon>0$. To understand this estimate it is useful to note that

$$
\operatorname{supp} \Sigma\left|\nabla X_{a}\right|^{2} \subset \bigcup_{\#(b) \geqq 3} \operatorname{supp} X_{b} .
$$

To prove (4.6) we observe that since $\Sigma\left|\nabla X_{a}\right|^{2}=0$ on the set $\left\{x \in X \mid \sum_{\#(a)=2} X_{a}^{2}(x)=1\right\}$, there is a $\delta$-neighborhood of this set and a number $0<\varepsilon_{2} \leqq \frac{1}{2}$ such that $\sum_{\#(a)=2} X_{a}^{2} \geqq 1-\varepsilon_{2}$ and $\Sigma\left|\nabla X_{a}\right|^{2} \leqq \varepsilon_{1}$ on this neighborhood and $1-\sum_{\#(a)=2} X_{a}^{2} \geqq \varepsilon_{2}$ outside of it. This implies (4.6) with $\varepsilon=\varepsilon_{1}\left(1-\varepsilon_{2}\right)^{-1}$ and $C_{\varepsilon}=\varepsilon_{2}^{-1} \sup \left(\Sigma\left|\nabla X_{a}\right|^{2}\right)$.

Inserting (4.5) and (4.6) into (4.1) and recalling (4.2) we arrive at (4.3). 
Now we pick $\tilde{X}_{a}$ so that

$$
|x|_{a} \geqq \text { const }|x| \text { on } \operatorname{supp} \tilde{X}_{a} .
$$

Lemma 4.5. Let $\Sigma_{1}=\min _{a} \inf H_{a} Q_{a}$. Fix positive $\delta<\Sigma_{1}-\Sigma$. Then the operators $B_{a}$ have only finite numbers of eigenvalues $<\Sigma$.

Proof. Each $C_{a}$ has a finite number of negative eigenvalues because of the restriction on $W_{a}$. Operators $D_{a}$ have finite numbers of eigenvalues $\leqq \Sigma$ because $\inf \sigma_{\text {ess }}\left(D_{a}\right)=\Sigma_{1}+\delta>\Sigma$. Since $B_{a}=C_{a} \oplus D_{a}$, the statement follows.

Lemma 4.6. The operators $H_{b}+I_{b} \tilde{X}_{b}-C_{\varepsilon}\langle x\rangle^{-2}$ with $\#(b) \geqq 3$ have finite numbers of eigenvalues $\leqq \Sigma$.

Proof. Since $I_{b} \tilde{X}_{b}-C_{\varepsilon}\langle x\rangle^{-2}$ is $H_{b}$-compact, the essential spectra of the operators under consideration equal $\sigma_{\text {ess }}\left(H_{b}\right)$. Since by the condition of Theorem 4.3, $\inf H_{b}>\Sigma$ for all $b$ with \# $(b) \geqq 3$, those operators have only finite numbers of eigenvalues $\leqq \Sigma$.

Lemma 4.7. Let each operator $F_{a}$ be self-adjoint with negative spectrum finite. Then $\Sigma X_{a} F_{a} X_{a}$ has a finite number of negative eigenvalues.

Proof. Let $\pi_{a}$ be the projection operator on the eigenspace of $F_{a}$ corresponding to the spectrum in $(-\infty, 0)$. Then

$$
\Sigma X_{a} F_{a} X_{a} \geqq \Sigma F_{a} \pi_{a} .
$$

Since each $\pi_{a}$ is finite dimensional, $\Sigma F_{a} \pi_{a}$ has a finite number of negative eigenvalues. Thus by the comparison theorem [RS3], $\Sigma X_{a} F_{a} X_{a}$ has only a finite negative spectrum.

Lemmas 4.4-4.7 imply via the comparison theorem the first statement of Theorem 4.3 (no symmetry). The proof with an allowance for symmetry is obtained by adding a few extra indices to the proof above (cf. Appendix 4).

Now we deduce Theorems 4.1 and 4.2 from Theorem 4.3.

Proof of Theorem 4.1. Writing $\left\langle\psi^{a}, I_{a} \psi^{a}\right\rangle$ and $\left\langle\psi^{a}, I_{a}^{2} \psi^{a}\right\rangle$ as sums of the convolutions of $V_{l}$ and $V_{l}^{2}$, respectively, $l \$ a$, with one-particle densities (see e.g. the proof of Theorem 4.2 below) and using the Young (or generalized Young) inequality [RS2] and the fact that $\psi^{a} \in L^{q}$ for any $q$, we find that $\left\langle\psi^{a}, I_{a} \psi^{a}\right\rangle \in L^{p} \cap L^{q}$ and $\left\langle\psi^{a}, I_{a}^{2} \psi^{a}\right\rangle \in L^{p / 2} \cap L^{r}$ with $p$ the same as in the statement and $\left.\infty \geqq q\right\rangle p$ and $\infty \geqq r \geqq p / 2$. Hence, since $p=v / 2$ and $\left\langle\psi^{a}, I_{a} \psi^{a}\right\rangle^{2}$ is positive, $W_{a}$ has only a finite number of bound states [RS3].

Before proceeding to the proof of Theorem 4.2, we recall the Hamiltonian of an ion with $N$ electrons and a nucleus of charge $Z$ (the masses are not restricted)

$$
H(N)=-\Delta-\sum_{i=1}^{N} \frac{Z}{\left|x_{i 0}\right|}+\sum_{i \neq j}^{1, N} \frac{1}{\left|x_{i j}\right|} .
$$

Here the nucleus is labeled by 0 , the electron charge is taken to be 1 and $X$ is, of course, the $v N$-dimensional hyperplane in $\mathbb{R}^{v(N+1)}$. The fact that the electrons are fermions is reflected in the restriction of $H(N)$ to functions of the permutationsymmetry types corresponding to one- and two-column Young tables. 
Next we note that in accordance with the HVZ theorem (Sect. 2 and Appendix 4) the bottom of the continuous spectrum of $H(N)$ (also if restricted to certain symmetry type functions) is defined only by two-cluster breakups of the form

$$
a_{s}=\{(0 \ldots \hat{s} \ldots N)(s)\}, \quad s=1, \ldots, N,
$$

i.e. by the one-electron ionizations. Here we assume that $H(N-1)$ has isolated eigenvalues. We conjecture that this always holds in the case when $H(N)$ has isolated eigenvalues (presently, it is known only for $N=Z+1$ ).

Finally, we mention that [RS3] the ground state, $\psi_{N}$, of $H(N)$ is unique and therefore rotationary invariant in the sense

$$
\psi_{N}(g x)=\psi_{N}(x) \text { with } g x=\left(g x_{1} \ldots g x_{N}\right) \text { and } g \in O(3)
$$

This is not, however, known for different permutation-symmetry types. If (4.8) fails to hold we use the fact that the ground states of the same symmetry type have the same parities [see Remark (b) to this section].

Proof of Theorem 4.2. To estimate $W_{a_{s}}$ we note that the intercluster interaction for $a_{s}$ is

$$
I_{a_{s}}=\sum_{i \neq 0, s} \frac{1}{\left|x_{i s}\right|}-\frac{Z}{\left|x_{0 s}\right|}
$$

and use standard screening estimates given in the two lemmas below. There we use the notations $y=x_{s}, I(y)=I_{a_{s}}$ and $\psi$ for the ground state of $H^{a_{s}}$ of a definite permutation symmetry type. We assume $\psi$ is rotationary invariant [in the sence of (4.8)]. If the latter fails to hold then we proceed as prescribed in Remark (b).

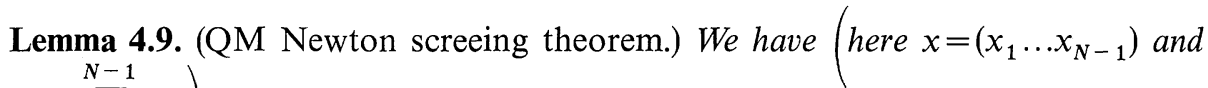
$\left.d x=\prod_{1}^{N-1} d x_{i}\right)$

$$
\langle\psi, I(y) \psi\rangle \geqq\left[N-1-Z-(N-1) \int_{\left|x_{1}\right| \geqq|y|}|\psi|^{2} d x\right]|y|^{-1} .
$$

Proof. First we compute in a standard way $(M=$ mass of the nucleus/mass of the electron)

$$
\langle\psi, I(y) \psi\rangle=(N-1) \int \frac{|\psi(x)|^{2}}{\left|x_{1}-y\right|} d x-Z \int \frac{|\psi(x)|^{2} d x}{\left|M^{-1} \Sigma x_{j}-y\right|} .
$$

Since $\psi$ is rotationary invariant [in the sence of (4.8)] the one-particle densities entering (4.11) are spherically symmetric. Hence by the Newton electrostatic theorem

$$
\int \frac{|\psi(x)|^{2}}{\left|x_{1}-y\right|} d x=|y|^{-1} \int_{\left|x_{1}\right| \leqq|y|}|\psi(x)| d x
$$

and similarly for the second integral on the right hand side of (4.11). Together with (4.11) this gives (4.10).

Lemma 4.10. (Simon.) The following estimate holds

$$
\left\langle\psi, I(y)^{2} \psi\right\rangle-\langle\psi, I(y) \psi\rangle^{2} \leqq \operatorname{const}(1+|y|)^{-4} .
$$

Proof. Using the elementary inequality

$$
|| a-\left.y\right|^{-1}-|y|^{-1}-\left.(a \cdot y)|y|^{-3}|\leqq 3| a\right|^{2}|y|^{-2}|a-y|^{-1},
$$


we obtain

$$
\left.|I(y)-(N-1-Z)| y\right|^{-1}+\left.\left(\sum_{i \neq 0, s} x_{i}-Z x_{0}\right) \cdot y|y|^{-3}|\leqq 3| y\right|^{-2} \sum_{i \neq s}\left|x_{i}\right|\left|x_{i}-y\right|^{-1} .
$$

Consider $\langle\psi, I(y) \psi\rangle$. Since $|\psi|^{2}$ is even, the integral, produced by the term $\left(\sum_{i \neq 0, s} x_{i}-Z x_{0}\right) \cdot y|y|^{-3}$, is zero. Using that $|\psi|^{2}$ falls off at infinity we estimate

$$
\left.|\langle\psi, I(y) \psi\rangle-(N-1-Z)| y\right|^{-1} \mid \leqq C(1+|y|)^{-3}
$$

(the dipole moment is zero for an even density). Similarly, we obtain

$$
\left.\left|\left\langle\psi, I(y)^{2} \psi\right\rangle-(N-1-Z)^{2}\right| y\right|^{-2} \mid \leqq C(1+|y|)^{-4} .
$$

These two inequalities imply the desired estimate.

These two lemmas and the fact that the bound states are exponentially bounded [see Remark (c) to Sect. 3] imply the inequality $\left(a=a_{s}\right)$

$$
W_{a}(y) \geqq(N-1-Z)|y|^{-1}-\operatorname{const}(1+|y|)^{-4} .
$$

So for $Z \leqq N-1$, the negative part of $W_{a}$ is short-range (the Newton screening theorem in QM). Hence $W_{a}$ have only finite number of bound states. This by virtue of Theorem 4.3 completes the proof.

\section{Nonexistence of Very Negative Ions}

Theorem. There is no very negative ions in $\mathrm{QM}$.

To give a mathematical formulation of the theorem we consider an $N$-electron ion with a nucleus of charge $Z$. For the sake of notational convenience we assume the nuclear mass to be infinite. In the units in which the electron charge and mass are 1 and $\frac{1}{2}$ respectively, the Schrödinger operator of such an ion is

$$
H_{N}=\sum_{i=1}^{N}\left(-\Delta_{i}-\frac{Z}{\left|x_{i}\right|}\right)+\sum_{i<j} \frac{1}{\left|x_{i}-x_{j}\right|} \text { on } L^{2}\left(\mathbb{R}^{v N}\right) \text {. }
$$

Here $\Delta_{i}$ stands for the Laplacian in $x_{i} \in \mathbb{R}^{v}$.

Ignoring the symmetry, the theorem asserts that

$$
H_{N} \geqq \inf \sigma_{\text {ess }}\left(H_{N}\right)=\inf H_{N-1} \text { for sufficiently large } N \text {. }
$$

[The second equality follows from the HVZ theorem (Sect. 2).] The symmetry can be taken into account in a routine simple way (see Appendix 4).

In this section we use a new partition of unity $\left\{K_{s}\right\}_{0}^{N}$ tailored for the problem. Let $x=\left(x_{1}, \ldots, x_{N}\right)$ and $|x|_{p}=N^{-1 / p}\left(\Sigma\left|x_{i}\right|^{p}\right)^{1 / p}$. We define $K_{s}$ by

$$
\operatorname{supp} K_{s} \subset\left\{\left.x \in \mathbb{R}^{v N}|| x\right|_{p} \leqq \gamma\left|x_{s}\right| \text { and }\left|x_{s}\right|>N^{-1 / p} \varrho\right\},
$$

where $\gamma>1$ is a given number, e.g. $\gamma=2$, for $s=1, \ldots, N$ and

$$
K_{0} \in C_{0}^{\infty}\left(\mathbb{R}^{v N}\right) \text { and } \operatorname{supp} K_{0} \subset\left\{x \in \mathbb{R}^{v N}|| x_{i} \mid<N^{-1 / p} \gamma \varrho\right\} \text {, }
$$

$K_{s}, s=1, \ldots, N$, is invariant under the action of the group $S_{N-1}^{(s)}$ and $K_{0}$, under the action of $S_{N}$. 
Here $S_{N}$ and $S_{N-1}^{(s)}$ are the groups of permutations of the indices $\{1, \ldots, N\}$ and $\{1, \ldots, N\} \backslash\{s\}$, respectively.

The localization formula with $\left\{K_{s}\right\}$ is

$$
H_{N}=\sum_{s=0}^{N} K_{s} H_{N} K_{s}-\sum_{s=0}^{N}\left|\nabla K_{s}\right|^{2}
$$

Proof. In each cone $K_{s}, s=1, \ldots, N$, we estimate (from below) the electrostatic interaction of the $s^{\text {th }}$ electron with the rest of the ion, using that $\sum_{i: i \neq s}\left|x_{i s}\right|^{-1} \geqq\left(N / \gamma+1-\frac{1}{2}\right)\left|x_{s}\right|^{-1}$ on $\operatorname{supp} K_{s}$ (this inequality is derived by evaluating the minimum of $\sum_{1}^{N}\left(1+a_{i}\right)^{-1}$ under the restrictions $a_{i} \geqq 0$ and $\sum_{1}^{N} a_{i}^{p} \leqq \gamma^{p} N$; here $\left.a_{i}=\left|x_{i}\right| /\left|x_{s}\right|\right)$ and that $H_{N-1}^{(s)} \geqq \Sigma_{N}$, where $H_{N-1}^{(s)}$ is the Hamiltonian of the ion without the $s^{\text {th }}$ electron:

$$
K_{s} H_{N} K_{s} \geqq\left(\Sigma_{N}+\left(N \mid \gamma+1-\frac{1}{2}-Z\right)\left|x_{s}\right|^{-1}\right) K_{s}, \quad \text { where } \quad \Sigma_{N}=\inf H_{N-1} .
$$

This estimate expresses a simple fact that this electron sees the nucleus shielded by the other electrons whose electrostatic repulsion prevails for sufficiently large $N$ (the classical Newton screening). In $K_{0}$ we estimate (again from below) the electrostatic repulsion between electrons using the fact that they cannot get more than $2 \gamma \varrho$ apart in $K_{0}$ and then we estimate the ground state energy of $N$ independent electrons by the classical Fermi method filling first $N / 2$ levels (counting the multiplicities) of the corresponding Dirichlet one-particle Hamiltonian with two electrons each (the Pauli principle!):

$$
K_{0} H_{N} K_{0} \geqq L_{N} K_{0}^{2} \text {, where } L_{N}=N(N-1) / 4 \gamma \varrho+\text { Fermi term. }
$$

The Fermi term is twice the sum of the first $N / 2$ eigenvalues (counting the multiplicities) of the one-particle Hamiltonian $-\Delta-Z /|x|$ on $L^{2}\left(B_{\gamma \ell}\right)$, where $B_{\varrho}=\left\{x \in \mathbb{R}^{v}|| x \mid \leqq \varrho\right\}$, with the Dirichlet boundary conditions. A good estimate of this term is tedious but its asymptotic behavior as $N \rightarrow \infty$ is easy to derive (using e.g. the hydrogen levels till the cutoff energy and a box model for the positive eigenvalues): it tends to $+\infty$ as $N \rightarrow \infty$.

Finally we estimate the localization error $-\Sigma\left|\nabla K_{s}\right|^{2}$ using an explicit construction of $\left\{K_{s}\right\}$ (see Appendix 3), (we set $p=N^{2}$ and absorb the factors $N^{1 / p}$ into the constants which otherwise are $p$-independent):

$$
\Sigma\left|\nabla K_{s}(x)\right|^{2} \leqq C N^{2 / 3}\left(|x|_{p}+\varrho\right)^{-2} .
$$

Putting together these inequalities and localization formula (5.4), we get

where

$$
H_{N} \geqq\left(L_{N}-C N^{1 / 2} \varrho^{-2}\right) K_{0}^{2}+\Sigma_{N}\left(1-K_{0}^{2}\right)+W,
$$

$$
W=\sum_{1}^{N}\left(N / \gamma+1-\frac{1}{2}-Z-C N^{1 / 2} \varrho^{-1}\right)\left|x_{s}\right|^{-1} K_{s}^{2}
$$

For $N$ sufficiently large and $\varrho \geqq 1$ we have that

$$
N \geqq \alpha Z \text { for some } \alpha>2 \text { implies } W \geqq 0 \text {. }
$$


Furthermore, since $L_{N}=O\left(N^{2} \varrho^{-1}\right)$ and $\Sigma_{N}$ does not grow as $N \rightarrow \infty$ we can choose $N$ so that $L_{N} \geqq \Sigma_{N}+C \varrho^{-2} N^{2 / 3}$. Together with the former inequality this implies $H_{N} \geqq \Sigma_{N}$.

\section{Appendix 1: Combes-Thomas Argument}

Combes-Thomas Argument (in the DHSV form) [CT, DHSV]. Let $\psi$ be an eigenfunction of $H$ with an eigenvalue $E, f$ a measurable function with the measurable weak derivative. Then

$$
e^{f} \psi \in L^{2}(X) \text { iff } E \notin \sigma_{\text {ess }}(H(i \lambda f) \quad \forall 0 \leqq \lambda \leqq 1 .
$$

Here, recall, $H(f)=(-i \nabla-\nabla f)^{2}+V$ with $V=\Sigma V_{l}$.

Sketch of the proof. Introduce the one parameter group of gauge transformations:

$$
U(\lambda): u \rightarrow e^{i \lambda f} u \text {. }
$$

The gauge-transformed Hamiltonian is given by

$$
H(\lambda f)=U(\lambda) H U(\lambda)^{-1} .
$$

We compute $H(f)=(-i \nabla-\nabla f)^{2}+V$. Hence the family $H(\lambda f)$ has an analytic continuation to $\lambda \in \mathbb{C}$. Applying the O'Connor projection lemma and the first Balslev-Combes theorem [RS3] one concludes that $\psi$ is $U(\lambda)$-analytic as long as $E$ stays away from $\sigma_{\text {ess }}(H(\lambda f))$. So $e^{f} \psi \in L^{2}(X)$ if $E \notin \sigma_{\text {ess }}(H(i \lambda f))$ for all $0 \leqq \lambda \leqq 1$.

\section{Appendix 2: An Inequality}

Proposition. Let $A$ be a densely defined closed operator on a Hilbert space such that $\operatorname{Re} A=\frac{1}{2}\left(A+A^{*}\right)$ and $\operatorname{Im} A=\frac{1}{2 i}\left(A-A^{*}\right)$ are defined and self-adjoint and $\operatorname{Im} A$ is ( $\operatorname{Re} A)$-bounded. Then

$$
\text { inf } \operatorname{Re} \sigma_{\text {ess }}(A) \geqq \inf \sigma_{\text {ess }}(\operatorname{Re} A) .
$$

The same inequality is also true for the full spectra.

Proof. Note first that $\operatorname{Re} A$ and $\operatorname{Im} A$ are self-adjoint by the assumptions. For any $\varepsilon>0$ there is an $n$-dimensional eigen-projection $P^{n}, n<\infty$, for $\operatorname{Re} A$ so that $T \equiv(\operatorname{Re} A)\left(\mathbb{1}-P^{n}\right) \geqq \inf \sigma_{\text {ess }}(\operatorname{Re} A)-\varepsilon$. The real spectrum of $A-(\operatorname{Re} A) P^{n}$ lies in $\left[\inf \sigma_{\text {ess }}(\operatorname{Re} A)-\varepsilon, \infty\right)$ as follows from the invertibility of

$$
T+i \operatorname{Im} A-\lambda=(T-\lambda)^{1 / 2}\left[\mathbb{1}+i(T-\lambda)^{-1 / 2}(\operatorname{Im} A)(T-\lambda)^{-1 / 2}\right](T-\lambda)^{1 / 2}
$$

for any $\lambda<\inf \sigma_{\text {ess }}(\operatorname{Re} A)-\varepsilon$. Since $\sigma_{\text {ess }}(A) \subset \sigma\left(A-(\operatorname{Re} A) P^{n}\right)$ by Weyl-type theorem of [S4, Theorem AI, 1], (*) follows. The second part of the proposition follows from the invertibility of $(* *)$ with $T=\operatorname{Re} A$.

\section{Appendix 3: Construction of $\left\{K_{s}\right\}$}

Let $\Omega_{s}=\left\{\left.x \in \mathbb{R}^{v N}|| x\right|_{p} \leqq\left|x_{s}\right|,\left|x_{s}\right| \geqq \varrho\right\}$ for $s=1, \ldots, N$ and $\Omega_{0}=\left\{\left.x \in \mathbb{R}^{v N}|| x\right|_{p} \leqq \varrho\right\}$. Then $\bigcup_{0}^{N} \Omega_{s}=\mathbb{R}^{v N}$. Let $\left\{F_{s}\right\}_{0}^{N}$ be a collection of real $C^{\infty}$-functions such that $F_{s}=1$ 
on $\Omega_{s}$. We define $K_{s}=F_{s}\left(\Sigma F_{i}^{2}\right)^{-1 / 2}$. So $\Sigma K_{i}^{2}=1$. Note that $\Sigma F_{i}^{2} \geqq 1$ since $F_{s}=1$ on $\Omega_{s} \forall s$ and $\bigcup \Omega_{s}=\mathbb{R}^{v N}$.

Now we construct $F_{s}$. Let $\chi \in C^{\infty}$ and $\chi(t)=1$ if $t \leqq 1$ and $=0$ if $t>1+\alpha$ for some fixed $\alpha>0$. Then $F_{s}(x)=\left(1-\chi\left(\left|x_{s}\right| N^{1 / p} / \varrho\right)\right) \chi\left(|x|_{p} /\left|x_{s}\right|\right)$ if $\left|x_{s}\right|>0$ and $=0$ if $\left|x_{s}\right|=0$ for $s=1, \ldots, N$ and $F_{0}(x)=\chi\left(|x|_{p} N^{1 / p} / \varrho\right)$. With this definition $K_{s}$ obeys (5.1)-(5.3). We show now that it obeys (5.5) as well.

First we prepare the ground for the estimation of $\sum\left|\nabla K_{s}\right|^{2}$. We compute

$$
\nabla K_{s}=\nabla F_{s}\left(\sum F_{j}^{2}\right)^{-1 / 2}-F_{s}\left(\sum F_{j} \nabla F_{j}\right)\left(\sum F_{j}^{2}\right)^{3 / 2} .
$$

So as vectors on $\mathbb{R}^{N+1}$ and $\mathbb{R}^{v(N+1) N}$

$$
\begin{aligned}
\|\nabla K\|^{2} & =\|\nabla F\|^{2}\|F\|^{-2}-|\langle F, \nabla F\rangle|^{2}\|F\|^{-4} \\
& \leqq\|\nabla F\|^{2}\|F\|^{-2} .
\end{aligned}
$$

Finally, we note the following simple inequalities [cf. (4.6)]

$$
\chi^{\prime 2} \leqq C_{1}\left(\varepsilon^{-1} \chi^{2}+\varepsilon\right) \text { and } \chi^{\prime 2} \leqq C_{1}\left(\delta^{-1}(1-\chi)^{2}+\delta\right),
$$

where $C_{1}$ is a constant depending only on $\alpha$. This can be derived using e.g. the explicit construction

$$
\chi(t)=2\left(\frac{t-1}{\alpha}\right)^{3}-3\left(\frac{t-1}{\alpha}\right)^{2}+1 \text { for } 1 \leqq t \leqq 1+\alpha .
$$

Now we proceed directly to an estimation of $\|\nabla F\|$. First we compute explicitly

$$
\begin{aligned}
\left|\nabla F_{s}\right|^{2}= & {\left[\varrho^{-1} \psi_{s}+\partial / \partial\left|x_{s}\right|\left(|x|_{p}\left|x_{s}\right|^{-1}\right) g_{s}\right]^{2} } \\
& +\left|x_{s}\right|^{-2} N^{-2 / p}\left(\sum_{i \neq s}\left|x_{i}\right|^{2(p-1)}\right)\left(\sum_{j}\left|x_{j}\right|^{p}\right)^{-\frac{2(p-1)}{p}} g_{s}^{2},
\end{aligned}
$$

where we have introduced the notation $\psi_{s} \equiv-\chi^{\prime}\left(\left|x_{s}\right| / \varrho\right) \chi\left(|x|_{p} /\left|x_{s}\right|\right)$ and $g_{s}=\left(1-\chi\left(\left|x_{s}\right| / \varrho\right)\right) \chi^{\prime}\left(|x|_{p} /\left|x_{s}\right|\right)$. Since $\left|x_{p} \leqq(1+\alpha)\right| x_{s} \mid$ on $\operatorname{supp} g_{s}$ and $\left|x_{s}\right|^{p-1}$ $\leqq\left(\sum\left|x_{j}\right|^{p}\right)^{\frac{p-1}{p}}$ we have $|\partial / \partial| x_{s}\left|\left(|x|_{p}\left|x_{s}\right|^{-1}\right)\right| \leqq\left(1+\alpha+N^{-1 / p}\right)\left|x_{s}\right|^{-1}$. Furthermore, using the inequalities $(a+b)^{2} \leqq 2 a^{2}+2 b^{2}$ and $\sum\left|x_{j}\right|^{2(p-1)} \leqq\left(\sum\left|x_{j}\right|^{p}\right)^{\frac{2(p-1)}{p}}$ we find

$$
\left|\nabla F_{s}\right|^{2} \leqq 2 \varrho^{-2} \psi_{s}^{2}+\left|x_{s}\right|^{-2}\left(2\left(1+\alpha+N^{-1 / p}\right)^{2}+N^{-2 / p}\right) g_{s}^{2} \text {. }
$$

Finally, since

$$
\begin{gathered}
|x|_{p} \leqq(1+\alpha)\left|x_{s}\right| \leqq(1+\alpha)^{2} \varrho \text { on } \operatorname{supp} \psi_{s} \\
\varrho \leqq\left|x_{s}\right| \text { and }|x|_{p} \leqq(1+\alpha)\left|x_{s}\right| \text { on } \operatorname{supp} g_{s}
\end{gathered}
$$

and, by virtue of (A.2),

$$
\psi_{s}^{2} \leqq C_{1}\left(\delta+\delta^{-1} F_{s}^{2}\right) \quad \text { and } \quad g_{s}^{2} \leqq C_{1}\left(\varepsilon+\varepsilon^{-1} F_{s}^{2}\right)
$$

we get taking $\delta=\varepsilon<1$

$$
\left|\nabla F_{s}\right|^{2} \leqq 24(1+\alpha)^{4} C_{1}\left(\varepsilon+\varepsilon^{-2} F_{s}^{2}\right)\left(\varrho+|x|_{p}\right)^{-2} N^{1 / p} .
$$

Summing up these inequalities we find

$$
\|\nabla F\|^{2} \leqq C_{2}\left(\varepsilon N+\varepsilon^{-2}\|F\|^{2}\right)\left(\varrho+|x|_{p}\right)^{-2} N^{1 / p},
$$


where $C_{2}=24(1+\alpha)^{4} C_{1}$, obviously $N$-independent. Picking here $\varepsilon=N^{-1 / 3}$ and recalling (A.1) we arrive at

$$
\sum_{1}^{N}\left|\left(\nabla K_{s}\right)(x)\right|^{2} \leqq C N^{1 / 2+1 / p}\left(|x|_{p}+\varrho\right)^{-2}
$$

with a $p$ - and $N$-independent constant $C$.

\section{Appendix 4: The Symmetry Groups}

Let $G$ and $G(a)$ be symmetry groups (or subgroups) of $H$ and $H_{a}$, respectively, and let $\sigma$ and $\alpha$ label the types of their irreducible representations. We always choose $G(a)$ to be a subgroup of $G$. The examples of $G(G(a))$ are trivial group, $S, S \times O(3)$ [trivial group, $\left.\bigotimes_{C_{i} \in a} S\left(C_{i}\right),\left(\bigotimes_{C_{i} \in a} S\left(C_{i}\right)\right) \otimes O(3)\right]$. Here $S$ and $S(C)$ are the groups of permutations of the identical particles in the whole system and in the cluster $C$, respectively. The trivial group, $\{\mathrm{id}\}$, is considered when we do not want to take the symmetry into account. Let $\alpha \prec \sigma$ express the fact that the irreducible representation $\alpha$ of $G(a)$ is present in the decomposition of the restriction of the irreducible representation $\sigma$ of $G$ to the subgroup $G(a)$. Finally, we denote by $H^{\sigma}$ and $H_{a}^{\alpha}$ the restrictions of $H$ and $H_{a}$ to the invariant subspaces on which the representations of $G$ and $G(a)$ are multiple to irreducible of the types $\sigma$ and $\alpha$, respectively. The projections on the invariant subspaces above will be denoted by $P^{\sigma}$ and $P_{a}^{\alpha}$. Then $H^{\sigma}=H P^{\sigma}=P^{\sigma} H$ and similarly $H_{a}^{\alpha}=H_{a} P_{a}^{\alpha}=P_{a}^{\alpha} H_{a}$.

To obtain the HVZ-theorem with the symmetry we note first that the $J_{a}$ can be constructed to commute with the symmetry groups $G(a)$.

Since $\sum_{\alpha \prec \sigma} P_{a}^{\alpha} P^{\sigma}=P^{\sigma}$ by the definition of the restriction $\alpha \prec \sigma$, the proof above applied to $H^{\sigma}$ yields that $\sigma_{\text {ess }}\left(H^{\sigma}\right) \subset \bigcup_{a, \alpha<\sigma} \sigma\left(H_{a}^{\alpha}\right)$. Together with the easy direction of which proof in the case of symmetry remains the same, this gives $\sigma_{\text {ess }}\left(H^{\sigma}\right)=\bigcup_{a, \alpha<\sigma} \sigma\left(H_{a}^{\alpha}\right)$, which is the HVZ theorem with symmetry.

Now we point out the allowances which have to be made in the proof of the theorem of Sect. 5 in order to take into account the symmetry consistently. Recall that $S_{N}$ and $S_{N-1}^{(s)}$ denote the groups of the permutations of $(1 \ldots N)$ and $(1 \ldots \hat{s} \ldots N)$, respectively. Let $P^{\sigma}$ and $P_{s}^{\alpha}$ be the projections on the subspaces on which the representations of $S_{N}$ and $S_{N}^{(s)}$ are multiple to irreducible of the types $\sigma$ and $\alpha$, respectively. Finally, let $\Sigma_{N}^{\sigma}=\inf \sigma_{\text {ess }}\left(H_{N}^{\sigma}\right)$. Then the obvious inequality $K_{s} H_{N-1}^{(s)} K_{s} \geqq \inf H_{N-1} K_{s}^{2}$ used to obtain (5.6) should be replaced by $P^{\sigma} K_{s} H_{N-1}^{(s)} K_{s} P^{\sigma} \geqq \Sigma_{N}^{\sigma} P^{\sigma} K_{s}^{2} P^{\sigma}$, wich follows readily if we use:

( $\alpha) \sum_{\alpha<\sigma} P_{s}^{\alpha} P^{\sigma}=P^{\sigma}$

$(\beta)$ invariance of $K_{s}$ with respect to permutation $S_{N-1}^{(s)}$ (so $K_{s}$ commutes with $\left.P_{s}^{\alpha}\right)$, and

$(\gamma) \Sigma_{N}^{\sigma}=\min _{\alpha<\sigma} \inf H_{N-1}^{\alpha}$ by the HVZ-theorem with the symmetry.

The second and last remark is about inequality (5.6): It should be placed between the $P^{\sigma}$ 's. 


\section{Remarks}

Section 2. Our proof of the HVZ theorem is related to that of Enss [E]. It is shorter than the latter since it uses the Weyl theorem while Enss' proof is based on the Weyl criterion (which is more powerful) and proves the Weyl theorem in its course. Besides, Enss' proof is more intuitive.

Section 3. (a) For almost all $x, a(x)=\{(1) \ldots(N)\}$, so $|\nabla f|^{2}<-E$, which gives the fastest possible decay.

(b) As was noticed by Agmon (see [CS]) the DHSV exponential bounds are equivalent to the Agmon optimal bounds.

(c) [DHSV] has shown that the DHSV $L^{2}$-exponential bound implies the DHSV $L^{p}$-exponential bound [i.e. $\left.e^{f} \psi \in L^{p}(X)\right]$.

(d) The references and comments on other works on the exponential fall off of bound states can be found in [DHSV, $\mathrm{H}-\mathrm{O}^{2} \mathrm{AM}$, and RS3] (we note only that the first general exponential bounds were obtained by O'Connor [OC]).

(e) The subspaces $\{x \in X \mid a(x)=a\}$ can be also written as ([SS])

$$
\left\{x \in X \mid x_{i}=x_{j} \text { iff } i \text { and } j \text { belong to the same cluster of } a\right\} \text {. }
$$

Section 4. (a) The Combes-Simon inequality can be given the following abstract form:

Lemma. Let $A$ and $P$ be self-adjoint operators with $R(P) \subset D(A)$ and $R(A) \subset D(P)$. Denote $Q=\mathbb{1}-P$. Then

$$
A \geqq P A P-P A Q W^{-1} Q A P+Q(A-W) Q
$$

for any invertible, positive operator $W$ for which the right hand side is defined.

Proof. The equality

$$
A=P A P+Q A Q+P A Q+Q A P
$$

and inequality

$$
|\langle Q A P \varphi, \varphi\rangle| \leqq \frac{1}{2}\left(\left\|U^{-1} Q A P \varphi\right\|^{2}+\left\|U^{*} Q \varphi\right\|^{2}\right),
$$

where $U$ is any invertible operator, resulting from the Schwartz inequality, imply (*) with $W=U U^{*}$.

(b) $H(N)$ has a finite number of eigenvalues on a subspace of any irreducible $S_{N^{-}}$or $S_{N} \times O(3)$-symmetry type or if the symmetry group is dropped altogether. Here $S_{N}$ is the group of permutations of $N$ indices (electrons). If the ground states of $H(N-1)$ of a certain $S_{N-1}$-symmetry type are not rotationary invariant [in the sence of (4.8)] as it is the case with the ground states of the $S_{N-1} \times O(3)$-symmetry types with nonzero total momenta, estimate (4.10) is not, in general, valid (the negative part of $\langle\psi, I(y) \psi\rangle$ does not fall off exponentially for $Z \leqq N-1$ ). However, since the ground states have same parity [the parity transformation is contained in $O(3)]$, |negative part of $\langle\psi, I(y) \psi\rangle \mid \leqq$ const $(1+|y|)^{-3}$ for $Z \leqq N-1$ as follows from inequality $(*)$ of the proof of Lemma 4.10 (the dipole moment is zero for even densities). Note that if the potentials are changed in such a way that the ground states have different parities or do not have definite parities at all (which might happen if there is an additional ("accidental") degeneracy (see [LL]), then the 
effective potential $\langle\psi, I(y) \psi\rangle$ has a dipole leading term $\left(|x|^{-2}\right.$ at infinity) which when sufficiently large might lead to the infinite number of bound states for $H(N)$.

(c) Note again that $\Sigma\left|\nabla X_{a}\right|^{2}$ falls off at infinity as $|x|^{-2}$, i.e. it behaves as a longrange $N$-body potential. This does not lead to the infinite number of bound states of $H$ in our case since $\Sigma\left|\nabla X_{a}\right|^{2}$ lives in the region of $X$ where the system splits into more than two clusters and therefore has the lowest threshold $>\Sigma=\inf \sigma_{\text {ess }}(H)$. If we remove the restriction on $\Sigma$, e.g. allow for three-cluster thresholds to be at $\Sigma$, then $\Sigma\left|\nabla X_{a}\right|^{2}$ is supported also in the region with the lowest threshold $\Sigma$. This, in our opinion (also, B. Simon, private communication) might account for the Effimov effect, the appearance of the infinite number of bound states in some short-range systems.

(d) One might try to use inequality (4.3) together with the comparison theorem [RS3] in order to obtain effective estimates of the number of bound states of $H$ along the lines of works [B1, B2, BF1, BF2, H1] (see also [G, WS, W]). Note that such estimates were given using different generalizations of the Birman-Schwinger principle by Yafaev [Y2], Klaus and Simon [KS], and Sigal [S2].

(e) The restriction on $\Sigma$, that it is defined only by two-cluster breakups, is a technical one. The essentially most general conditions under which the finiteness of $\sigma_{d}(H)$ is still true were given in [S1]. To obtain this result by the method presented here one might try to counter $-\Sigma\left|\nabla X_{a}\right|^{2}$ with $\varepsilon(-\Delta)$ borrowed from $-\Delta$.

(f) Theorem 4.2 can be obviously generalized to include molecular ions.

(g) In the case $Z=N-1$ the proof of Lemma 4.10 is especially simple. Indeed, by virtue of

$$
|| a-\left.y\right|^{-1}-\left.|y|^{-1}|\leqq| a|| y\right|^{-2}\left(1+|a||a-y|^{-1}\right.
$$

we get

$$
\begin{aligned}
|I(y)| & \leqq \sum_{i \neq 0, s}|| x_{i}-\left.y\right|^{-1}-\left|x_{0}-y\right|^{-1} \mid \\
& \leqq|y|^{-2} \sum_{i \neq s}\left|x_{i}\right|\left(1+\left|x_{i}\right|\left|x_{i}-y\right|^{-1}\right),
\end{aligned}
$$

which, along with the fact that $|\psi|^{2}$ is bounded and fast decreasing, implies that $\left\langle\psi, I(y)^{2} \psi\right\rangle \leqq C(1+|y|)^{-4}$.

Section 5. (a) Different generalizations (not discussed here) of the theorem of this section are possible. Among them we list the extensions of this theorem to molecules and to non-Coulomb potentials (cf. [R]).

(b) The idea of using $|x|_{p}$ with $p<\infty$ comes from Ruskai [R]. (Originally the author used the $K_{s}$-partition with $p=\infty$.)

(c) This section has many common points with Ruskai [R] and Zhislin $[\mathrm{Z} 1, \mathrm{Z} 2]$.

Appendix 3. (a) We used the same function $\chi$ in different, independent constructions. The reason for this is our laziness.

(b) We believe that the estimate (A.4) can be improved considerably: Our conjecture is that $\sum_{s=0}^{N}\left|\left(\nabla K_{s}\right)(x)\right|^{2} \leqq C\left(1+\Sigma \varphi_{s}(x)\right)\left(|x|_{p}+\varrho\right)^{-2}$, where $\varphi_{s}=\left(\left|\psi_{s}\right| /\left\|\psi_{s}\right\|_{\infty}\right)^{2}$, is true. Note that (A.3) implies that $\operatorname{supp} \varphi_{s} \subset\left\{x \in \mathbb{R}^{3 N}|| x_{j} \mid<\gamma^{2} \varrho \forall i\right\}$. The factor $N^{1 / 2}$ should, in our opinion, disappear due to a compensation in $\|\nabla F\|^{2}-|\langle F, \nabla F\rangle|^{2} /\|F\|^{2}$. 
Acknowledgements. It is a pleasure to thank S. Agmon, W. Hunziker, Y. Kannai, E. Lieb, and B. Simon for fruitful discussions; E. Lieb and B. Simon for turning our attention to the problems of very negative ions and negative ions, respectively; M. B. Ruskai for explaining her results to me, and J. Morgan III for reference comments. Moreover, critical remarks of B. Simon motivated improving the partition of unity in Sect. 5 .

\section{References}

[A] Agmon, S. : Jerusalem, preprint 1980

[AZS] Antonets, M.A., Zhislin, G.M., Shereshevskii, J.A.: Theor. Math. Phys. 16, 800-808 (1972)

[B1] Bazley, N.W.: Proc. Natl. Acad. Sci. USA 45, 850-853 (1959)

[B2] Bazley, N.W.: Phys. Rev. 120, 144-149 (1960)

[BF1] Bazley, N.W., Fox, D.W.: Phys. Rev. 124, 483-492 (1961)

[BF2] Bazley, N.W., Fox, D.W.: J. Res. Nat. Bur. Standards, Sect, B65, 105-111 (1961)

[C] Combes, J.M.: In: Rigorous atomic and molecular physics. Velo, G., Wightman, A.S., (eds.) New York: Plenum 1981

[CS] Carmona, R., Simon, B. : Commun. Math. Phys. 80, 59-98 (1981)

[CT] Combes, J.M., Thomas, L.: Commun. Math. Phys. 34, 251-270 (1973)

[DHSV] Deift, P., Hunziker, W., Simon, B., Vock, E. : Commun. Math. Phys. 64, 1-34 (1978)

[E] Enss, V.: Commun. Math. Phys. 52, 233-238 (1977)

[G] Gould, S.H.: Variational methods for eigenvalue problems. Toronto: Univ. of Toronto Press, 1966

[Hi] Hill, R.N. : J. Math. Phys. 18, 2316-2330 (1977)

[H] Hunziker, W.: Helv. Phys. Acta 39, 451-462 (1966)

[H-O $\left.{ }^{2} \mathrm{AM}\right]$ Hoffmann-Ostenhof, M., Hoffman-Ostenhof, T., Ahlrichs, R., Morgan III, J.: Mathematical problems in theoretical physics. In: Lecture Notes in Physics Vol. 116. Berlin, Heidelberg, New York: 1980, 62-67

[I] Ismagilov, R. : Sov. Math. Dok1. 2, 1137-1140 (1961)

[K] Kato, T.: (unpublished)

[KS] Klaus, M., Simon, B.: Commun. Math. Phys. 78, 153-168 (1980)

[LL] Landau, L.D., Lifshitz, E.M.: Quantum mechanics. London, New York: Pergamon Press 1977

[L] Lieb, E.H.: Rev. Mod. Phys. 48, 553-569 (1976)

[M] Morgan III, J. : J. Operat. Theor. 1, 109-115 (1979)

[MS] Morgan III, J., Simon, B.: Int. J. Quant. Chem. 17, 1143-1166 (1980)

[OC] O'Connor, T.: Commun. Math. Phys. 32, 319-340 (1973)

[R] Ruskai, M.B.: Commun. Math. Phys. 82, 457-469 (1982)

[RS1] Reed, M., Simon, B.: Methods of modern mathematical physics. New York: Academic Press 1972

[RS2] Reed, M., Simon, B.: Methods of modern mathematical physics, II. New York: Academic Press 1975

[RS3] Reed, M., Simon, B. : Method of modern mathematical physics, IV. New York: Academic Press 1978

[S1] Sigal, I.M.: Commun. Math. Phys. 48, 137-154 (1976)

[S2] Sigal, I.M.: Duke Math. J., (submitted)

[S3] Sigal, I.M. : Proceedings of the VI ${ }^{\text {th }}$ International Conference on Mathematical Physics (W. Berlin, August 11-20, 1981). In: Lecture Notes in Physics. Berlin, Heidelberg, New York: Springer 1981

[SS] Sigalov, A.G., Sigal, I.M.: Theor. Math. Phys. 5, 990-1005 (1970)

[Sim1] Simon, B.: Commun. Math. Phys. 55, 259-274 (1977)

[Sim2] Simon, B.: Quantum mechanics for Hamiltonians defined as quadratic forms, Princeton, N.J. : Princeton Univ. Press 1970

[U] Uchiyama, J.: Publ. Res. Inst. Math. Sci., Kyoto Univ. A, 5, 51-63 (1969)

[VW] van Winter, C.: Danske V.d. Selsk. Mat.-Fys. Skr. 2, 1-60; 1-94 (1964-1965)

[WS] Weinstein, A., Stanger, W.: Methods of intermediate problems for eigenvalues. New York: Academic Press 1972 
[W] Weinberger, H.F.: Variational methods for eigenvalue approximation, SIAM, Philadelphia, 1974

[Y1] Yafaev, D.R.: Math. USSR, Izv. 10, 861-896 (1976)

[Y2] Yafaev, D.R.: Theor. Math. Phys. 27, 328-343 (1977)

[Z1] Zhislin, G.M.: Tr. Mosk. Mat. Obs. 9, 81-128 (1960) (in Russian)

[Z2] Zhislin, G.M.: Theor. Math. Phys. 7, 571-578 (1971)

[Z3] Zhislin, G.M.: Theor. Math. Phys. 21, 971-980 (1974)

[Z4] Zhislin, G.M.: Usp. Mat. N. 16, 149-152 (1961) (in Russian)

[ZV] Zhislin, G.M., Vugalter, S.A.: Theor. Math. Phys. 32, 602-614 (1977)

Communicated by B. Simon

Received December 18, 1981; in revised from March 9, 1982

Note added in proof. The technique above is effective within the framework of Agmon's method of proving the exponential bounds. More specifically, the localization formula $H=\Sigma\left(X_{a} H X_{a}-\left|\nabla X_{a}\right|^{2}\right)$ implies $H \geqq \omega+I$, where $\omega=\Sigma\left(\inf H_{a}\right) X_{a}^{2}$ and $I=\Sigma\left(I_{a} X_{a}^{2}-\left|\nabla X_{a}\right|^{2}\right)$. Since $I$ is $H$-compact, $\chi_{R} I(H+1)^{-1}$ $\rightarrow 0$ as $R \rightarrow \infty$ for bounded $C^{2}$ functions $\chi_{R}$ with supp $\chi_{R} \subset\{|x|>R\}$. So for any $\varepsilon>0$ there is $R$ s.t. $\chi_{R} H \chi_{R} \geqq[\omega-\varepsilon(H+a)] \chi_{R}^{2}$, where $a=\inf H+1$. The latter inequality plays an important role in Agmon's method [together with the elementary inequality $\left\langle\left(H-E-\left|\nabla f^{\prime}\right|^{2}\right) e^{f} \chi_{R} \psi, e^{f} \chi_{R} \psi\right\rangle \leqq M\left\|e^{f} \chi_{R} \psi\right\|$, where $M=\left\|e^{f} \Delta \chi\right\|\|\psi\|+2\left\|e^{f} \nabla \chi\right\|\|\nabla \psi\|$, valid for any bounded $C^{2}$ function $f$ and an eigenfunction $\psi$ of $H$ with the eigenvalue $E$, it implies validity the DHSV bound (see Sect. 3)]. 